\title{
GLAD!
}

Revue sur le langage, le genre, les sexualités

$05 \mid 2018$

Raconter les sexualités depuis la marge

\section{Dracula : mécanique des fluides et roman des angoisses circulatoires}

Bram Stoker's Dracula: Fluid Mechanics and Circulation Trouble

\section{Maël Baussand}

\section{(2) OpenEdition}

\section{Journals}

Édition électronique

URL : http://journals.openedition.org/glad/1248

ISSN : 2551-0819

Éditeur

Association GSL

\section{Référence électronique}

Maël Baussand, «Dracula : mécanique des fluides et roman des angoisses circulatoires », GLAD! [En ligne], 05 | 2018, mis en ligne le 15 décembre 2018, consulté le 17 décembre 2020. URL : http:// journals.openedition.org/glad/1248

Ce document a été généré automatiquement le 17 décembre 2020.

\section{c) $($ ) $\$$}

La revue GLAD! est mise à disposition selon les termes de la Licence Creative Commons Attribution Pas d'Utilisation Commerciale - Pas de Modification 4.0 International. 


\title{
Dracula : mécanique des fluides et roman des angoisses circulatoires
}

\author{
Bram Stoker's Dracula: Fluid Mechanics and Circulation Trouble
}

\author{
Maël Baussand
}

Que l'importance du sang soit emblématique dans Dracula (1897), voilà qui ne manquera pas de faire consensus. Mais il existe peu, voire aucune entreprise visant à démontrer que la véritable "mécanique des fluides " pensée par Bram Stoker dépasse l'apparition seule du sang, pour s'avérer être une mise en scène d'angoisses de nature circulatoire - c'est-à-dire liées aux fluides corporels.

2 Définie comme étant le mouvement propre à un fluide en circuit fermé, la " circulation ", pour les besoins de notre analyse, étendra sa définition à la provocation contrôlée (ou non) d'un écoulement. De fait, un roman tel que Dracula semble devoir en constituer l'interprétation la plus fortement chargée. La « mécanique des fluides » chez Stoker demande de prendre un angle de recherche inédit: celui du trouble profond généré par l'apparition, la manipulation, et l'usage symbolique des fluides corporels, générateur de ces angoisses circulatoires ${ }^{1}$. Car c'est en fait tout le système d'« échange de fluides » qui est signifiant, et même performatif, dans le roman. Par une étrange coction, c'est-à-dire une correspondance fongible des humeurs qui rappelle les hypothèses d'Aristote ${ }^{2}$, le fluide "sang " dans Dracula est incidemment lui-même de nature «fluide». Il prend tour à tour allure de sang des règles (ou, pourquoi pas, de sang de la défloration); de sperme (la charge érotique du texte n'étant plus à prouver); et de lait (le vampire "s'allaite", ou boit, au cou de ses victimes, mais dans les dérivations sémantiques du mythe il faut également boire le sang du monstre pour renaître vampire).

3 Or, la grande cohérence symbolique de cette correspondance existant entre ces trois fluides corporels, dans les discours sociaux et médicaux, en fait les fluides essentiels de la différence sexuelle - des fluides vitaux, génitaux et sexuels, mais aussi sexués et genrés ${ }^{3}$. Salvatore D'Onofrio écrit ainsi, dans Les fluides d'Aristote. Lait, sang et sperme dans l'Italie du Sud (2014) ${ }^{4}$, que " lait, sang et sperme, maintiennent et reproduisent la vie, mais sont aussi les substances que toutes les cultures humaines manipulent, du point de 
vue symbolique ». C'est ici toute une vérité des mythes et l'importance signifiante de nos représentations qu'il met en évidence. "Une sécrétion, on le sait, c'est ce qui sépare, discerne, dissocie, dissout le lien, tient au secret ", écrit Jacques Derrida dans Voiles (1998) $)^{5}$. Il montre ainsi que les fluides corporels, du signe à l'indice, du secret à l'énigme, font office de symboles (au sens étymologique littéral, qui signifie «jeter ensemble ») : ils invitent à les déchiffrer, leurs manifestations constituent un langage codé. Il s'agira donc bien de reconstruire le lien signifiant/signifié permettant d'établir une reconnaissance, laquelle fait de la «circulation» elle-même le cœur de nos préoccupations.

4 Quelles sont alors les conséquences de la manipulation de tels fluides, aussi denses, aussi chargés, dans Dracula? Dans la mesure où la thématique des fluides, par l'esthétique du trouble ${ }^{6}$ qu'elle convoque, possède une grande force de confiscation de l'attention, l'écriture devient un exercice esthétique exigeant, qui ne peut donc pas, dès lors, se prétendre neutre. La mise en scène gothique des angoisses, dans l'écriture, passe ainsi principalement par cette métaphore circulatoire, dont le roman se fait un vecteur de purgation, voire d'exorcisme. Il va donc bel et bien falloir parler « échanges de fluides ", au sens littéral du terme, avec toute la charge affective que l'expression implique.

\section{Blood Is Thicker Than Water ${ }^{7}$ : fluides corporels et angoisse sexuelle}

\section{Topique féminine du «trop de fluides » : la menace du débordement}

5 Dans Dracula, le monstre, figure de la limite, est un bouleversement, un trouble suprême, l'incarnation de cette menace venue des marges - corporelles, mais aussi géographiques - dont les femmes seront les victimes impuissantes. Le vampire exprime, répand précisément ce qui était intime, subsumé à une intériorité cachée. Il fait s'écouler les fluides et provoque ainsi une peur viscérale, un défoulement de passions. Là réside la clef de lecture principale, véritablement cathartique, selon laquelle il convient d'analyser ses attaques. L'action narrative se déroule au sein de classes sociales dominantes, bourgeoises, lesquelles sont très strictement préoccupées par la prévention de tout «débordement" - étrange écho à cette ère d'industrialisation massive où le contrôle même de l'eau représente un enjeu social essentiel. Ce choix du cadre narratif est loin d'être anodin: contrôler l'eau, à l'époque du roman, s'accompagne directement de stratégies de contrôle du corps, et plus particulièrement encore du corps féminin ${ }^{8}$.

6 En effet, selon les héritiers de la pensée aristotélicienne, comme Galien et Hippocrate, on observe une constellation symbolique, véritablement topique, qui relie le fluide, le liquide, l'eau, aux femmes. Là où la femme est froide et humide, l'homme est chaud et sec. Là où elle est changeante, imprévisible et superficielle (shallow en anglais, qui est incidemment aussi le mot utilisé pour décrire des eaux peu profondes), il est solide, stable et droit. Cette opposition femme/molle/eau/humide/froid et homme/dur/feu/ $\mathrm{sec} /$ chaud correspond à la relecture, opérée par le roman, de la société industrielle qui consacre, contre ce qu'elle tend à maîtriser (l'eau d'abord, et plus largement les éléments naturels et les femmes), la force $\mathrm{du}$ feu, de la technique et des hommes (fourneaux, charbon, usines...). À la femme échoit ainsi l'instabilité liquide et ses 
profondeurs troubles. Mais, face à la cohérence topique existant entre liquidité et féminité, il importe, d'un point de vue critique, de souligner la spécificité des fluides corporels face à cette fluidité générique. La figure de l'hydropique est ainsi

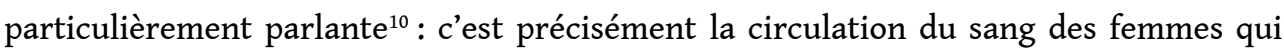
pose des enjeux de santé très prégnants dans la littérature, d'abord médicale mais pas seulement, et ce depuis l'Antiquité, avec la mise en place de dispositifs visant à son bon écoulement en particulier. Ceci génère une angoisse circulatoire chez les femmes ellesmêmes, ainsi sommées de maîtriser ces fluides liés à la reproduction comme à la sexualité $\left.{ }^{11}\right)$. Car ce rapprochement des femmes avec l'eau dans la perspective médicale fait d'elles des vecteurs particuliers censés transmettre des maladies de pléthore, dues à la surabondance de fluides que ne peut réguler la coction des humeurs. Ce discours avait des conséquences sociales d'une redoutable concrétude pour les femmes et leur «trop de fluides ». Trop " froides » effectivement ${ }^{12}$, les femmes devaient se "purger " régulièrement, par les règles et les grossesses, ou par des saignées et lavements divers.

7 La figure du vampire apparaît donc précisément comme une sorte de purge presque vengeresse, qui aurait mal tourné : en faisant des femmes les victimes toutes désignées du monstre, Bram Stoker ne se contente pas seulement de reproduire les croyances sociales misogynes de son époque, à l'échelle de son roman - il les transfere, en les faisant circuler dans le texte. Dans Dracula, le rôle des personnages féminins est justement de bouleverser, par la menace constante de débordement qu'elles représentent - une menace que la figure du vampire rendra manifeste. Le corps dans Dracula, et plus particulièrement encore le corps féminin, n'est donc pas une enveloppe fermée ni la peau une ligne close et rassurante: les deux sont déjà ouverts, et potentiellement à même de déborder. Et, puisque, selon Thomas Laqueur ${ }^{13}$ (qui a su montrer l'importance de la reconsidération symbolique des diverses sécrétions corporelles, et plus particulièrement génitales), on considère partout, sans cesse, que le mâle est l'étalon du genre humain, la femme devient une étrangeté fondamentale. Si l'homme est le modèle par excellence, la référence définitive, la femme ne peut donc être perçue, dans ce système, que comme le premier Autre, peut-être même le " premier monstre $^{14}{ }^{1}$ ( (qu'est-ce que le monstre, sinon une altérité radicale ?).

8 L'intérêt de la présence monstrueuse dans Dracula est ainsi rendu explicite: intouchable et tabou, le vampire renvoie directement à ce qui trouble effectivement dans l'écoulement visible des fluides ${ }^{15}$. Les attaques de Dracula seront en effet codifiées par deux modalités "débordantes" précises, directement reliées aux apparitions de fluides : le tabou et la souillure. Cela n'a rien d'étonnant si, à l'instar de Mary Douglas, nous estimons que le corps est le lieu privilégié de toute conceptualisation de la souillure, en plus d'être le modèle par excellence de tout système fini :

La souillure n'est jamais un phénomène isolé. (...) Les notions de pollution n'ont de sens que dans le contexte d'une structure totale de la pensée dont la clé de voûte, les limites, les marges et les cheminements internes, sont liés les uns aux autres $(. . .)^{16}$.

9 Ainsi, « la matière issue [des] orifices est de toute évidence marginale. Crachat, sang, lait, urine excréments, larmes, dépassent les limites du corps du fait même de leur sécrétion. (...) L'erreur serait de considérer les confins du corps comme différents des autres marges ${ }^{17}$. »

10 Mais la coction narrative de trois fluides sexuels en particulier dit quelque chose de la relation de la société victorienne à la sexualité. 


\section{Angoisse de l'acte sexuel : une peur dévorante}

11 L'une des fonctions du roman gothique est la mise en scène des peurs profondes ${ }^{18}$. Il existe à cet égard, dans l'écriture de Stoker, un lien stable et cohérent entre cette angoisse de nature circulatoire et une angoisse purement sexuelle. Les attaques du vampire, point culminant de tension narrative, remplissent une fonction jubilatoire car impudique. Elles répondent à l'appétit haptique de l'œil et à sa pulsion de (sa)voir ${ }^{19}$. Dans cette perspective, on ne peut donc pas faire l'économie d'aborder dans le roman le cas des fluides génitaux et sexuels. Il faut se représenter une nouvelle fois ce que peut avoir de bouleversant et de brutal, dans le contexte d'une société aussi répressive, la pleine révélation de ces fluides que l'on cache, et des manifestations ouvertement sexuelles qui leur sont liées ${ }^{20}$.

12 En cultivant cette "esthétique du trouble» par la plasticité du sang qui opère différentes transformations humorales (tour à tour sperme, lait, menstrues), l'écriture de Stoker manifeste un potentiel fort de fascination ${ }^{21}$, ou, pour reprendre les mots mêmes du roman (voir ci-dessous), "une volupté à la fois émouvante et repoussante ". Il faut également rajouter à cela le dégoût général éprouvé pour tout ce qui concerne l'intérieur du corps, universel: s'y mêlent allègrement l'effroi et l'émoi du corps ouvert, impacté dans son intimité. Le lien entre sexualité et intimité, exploité dans le roman, est ainsi clair: les attaques du monstre sont précisément violentes parce qu'elles mettent en scène l'abolition abrupte des frontières corporelles et sociales, dans une logique de dévoilement, de déchirement au point de choc, de heurt. Mais ce que manifeste prioritairement l'écriture de Stoker, à chaque attaque vampirique, c'est un fantasme très littéral de dévoration/consommation, où la bouche du vampire devient organe sexuel de prédilection :

\begin{tabular}{|l|l|}
\hline & Je n'osais relever les paupières, \\
I was afraid to raise my & mais je continuais néanmoins à \\
eyelids, but looked out and saw & regarder à travers mes cils, et je \\
perfectly under the lashes. The & voyais parfaitement la jeune \\
girl went on her knees, and & femme, maintenant agenouillée, \\
bent over me, simply gloating. & de plus en plus penchée sur moi, \\
There was a deliberate & l'air ravi, comblé. Sur ses traits \\
voluptuousness which was & était peinte une volupté à la fois \\
both thrilling and repulsive, & émouvante et repoussante et, \\
and as she arched her neck she & tandis qu'elle courbait le cou, elle \\
actually licked her lips like an & se pourléchait réellement les \\
animal, till I could see in the & babines comme un animal, à tel \\
moonlight the moisture & point que je pus voir à la clarté \\
shining on the scarlet lips and & de la lune la salive scintiller sur \\
on the red tongue as it lapped & les lèvres couleur de rubis et sur \\
the white sharp teeth ${ }^{22}$. & la langue rouge qui se promenait \\
& sur les dents blanches et \\
& pointues
\end{tabular}

13 Dans cet extrait particulièrement parlant, premier acte proprement vampirique du roman, le personnage de Jonathan Harker est la proie consentante de trois femmes, vampires et donc prédatrices, dans le château du comte. La scène est à la fois cauchemardesque, et d'une sensualité hyperesthésique - tous les sens y sont mobilisés. On remarque une surabondance de détails signifiants : l'ambiguïté toute évocatrice des 
"lèvres rouges", et cette "salive qui scintille " connotant la présence de fluides sexuels. Cette surcharge descriptive véhicule immédiatement une sensation hypnotique, qui communique la transe dans laquelle se trouve Jonathan. Le sous-texte est presque trop évident: Jonathan serait-il sur le point d'être engagé dans une fellation, acte pour lequel sa partenaire tirerait presque plus de plaisir que lui? Plus largement, la bouche et ses lèvres peuvent être rapprochées des lèvres vaginales. La vulve est elle aussi porteuse d'un grand appétit (sexuel) dans diverses représentations médicales, érotiques et littéraires - un appétit qu'il aura fallu contrôler par le discours notamment médical ${ }^{24}$ - où le mythe du " vagina dentata » prend ainsi tout son sens.

\begin{tabular}{|c|c|}
\hline $\begin{array}{l}\text { Lower and lower went her } \\
\text { head as the lips went below } \\
\text { the range of my mouth and } \\
\text { chin and seemed to fasten on } \\
\text { my throat. Then she paused, } \\
\text { and I could hear the churning } \\
\text { sound of her tongue as it } \\
\text { licked her teeth and lips, and I } \\
\text { could feel the hot breath on } \\
\text { my neck. Then the skin of my } \\
\text { throat began to tingle as one's } \\
\text { flesh does when the hand that } \\
\text { is to tickle it approaches } \\
\text { nearer, nearer. I could feel the } \\
\text { soft, shivering touch of the } \\
\text { lips on the super sensitive } \\
\text { skin of my throat, and the } \\
\text { hard dents of two sharp teeth, } \\
\text { just touching and pausing } \\
\text { there. I closed my eyes in } \\
\text { languorous ecstasy and } \\
\text { waited, waited with beating } \\
\text { heart }{ }^{25} \text {. }\end{array}$ & $\begin{array}{l}\text { Sa tête descendait de plus en plus, } \\
\text { ses lèvres furent au niveau de ma } \\
\text { bouche, puis de mon menton, et } \\
\text { j'eus l'impression qu'elles allaient } \\
\text { se refermer sur ma gorge. Mais } \\
\text { non, elle s'arrêta et j'entendis un } \\
\text { bruit, un peu semblable à un } \\
\text { clapotis [nous soulignons], que } \\
\text { faisait sa langue en léchant encore } \\
\text { ses dents et ses lèvres tandis que } \\
\text { je sentais le souffle chaud passer } \\
\text { sur mon cou. Alors la peau de ma } \\
\text { gorge réagit comme si une main } \\
\text { approchait de plus en plus pour la } \\
\text { chatouiller, et ce que je sentis, ce } \\
\text { fut la caresse tremblante des } \\
\text { lèvres sur ma gorge et la légère } \\
\text { morsure de deux dents pointues. } \\
\text { La sensation se prolongeant, je } \\
\text { fermai les yeux dans une extase } \\
\text { langoureuse. Puis j'attendis - } \\
\text { j'attendis, le cœur battant }{ }^{26} \text {. }\end{array}$ \\
\hline
\end{tabular}

"Sucer » ou "lécher » est une implication intime et sensuelle de la bouche comme organe aspirant voire dévorant, laquelle est évidemment lourde de sous-entendus. Quand le monstre se nourrit, c'est un acte sexuel à peine déguisé, mais aussi un acte primitif, bestial, où la faim et le désir se confondent. Le «terrible plaisir » éprouvé par Jonathan Harker met aussi en scène une libération certaine : après s'être attardée dans l'ombre de la répression, la sexualité émerge à la claire lumière du discours social victorien, dont le roman se fait la mimesis fallacieuse ${ }^{27}$.

15 Il serait donc bon, à cet égard, d'examiner cet organe même qui semble en être le point de jonction: outre la bouche dévoratrice, ce sont les dents du vampire, en tant qu'instrument de circulation et de transfusion (des outils filtrant le fluide et le transmettant au besoin), qui seront beaucoup décrites tout au long du roman. Cette dentition inhumaine est la source même des angoisses car elle rappelle sans cesse, dans le roman, que la peau n'est pas une ligne fermée : à tout moment, le monstre peut transpercer l'enveloppe charnelle pour laisser s'échapper les fluides. Ainsi « canines » pour rappeler l'homme ("sharp white teeth ») ou « crocs » pour rappeler la bête (" canine teeth»), les dents du vampire entretiennent cette indistinction fondamentale du monstre, sa nature hybride essentielle, fluide, qui est précisément ce qui le rend monstrueux. Ces canines, ou crocs, sont ici visiblement rétractables, et ne sortent 
qu' 'à propos ", sous le coup d'émotions violentes (désir ou excitation puissants, colère intense, faim dévorante... la limite entre chacune étant, nous l'avons dit, elle-même fluide, floue, et cette indifférenciation soigneusement cultivée). Qu'en conclure, sinon que les crocs du vampire sont aussi un organe érectile? Deux petits pénis qui pénètrent la chair, et déchirent la peau pour faire couler le sang. C'est ici le mythe de l'hymen et de la virginité cent fois répété dans le roman - malgré de très nombreuses scènes homo-érotiques, les femmes restent les victimes principales du vampire. Faut-il donc voir le roman comme une gigantesque métaphore, une sorte de "conte pour adultes", qui se fait l'expression nécessaire, exorcisée, de la « chose qui trouble »?

\section{Une angoisse combinée du féminin-fluide et du sexe : l'angoisse de la sexualité féminine}

16 Ce que fait la femme de son corps semble être une question préoccupante. Mais le XIXe siècle passe également comme étant le grand siècle par excellence de la répression féminine $^{28}$. Ce "siècle de l'hystérie ${ }^{29}$ " a fait d'une affliction, utérine jusque dans son étymologie, le symptôme de cet état de fait. Le roman semble d'ailleurs être, à bien des égards, une mise en scène du positivisme scientifique de Charcot. Cette "peur hystérique» de la sexualité féminine s'incarne dans le roman en deux avatars féminins : les personnages de Mina et de Lucy - lesquelles en exemplifient chacune un aspect. Car «l'hystérie » de Lucy et Mina se définit plus particulièrement face à un phénomène qui, symboliquement plus qu'ontologiquement, semble devoir marquer le début de la sexualité féminine - ou, à défaut, de la maturité sexuelle d'une femme : la menstruation. C'est une perspective évidemment très cissexiste, mais il est juste de dire que la menstruation, dans son acceptation sémantique, ne s'est guère construite autrement que comme une affliction « typiquement féminine ${ }^{30}$ ».

17 Marie Mulvey-Roberts, professeure associée à l'université de Bristol, dont la spécialité recouvre l'analyse des fluides corporels dans la littérature gothique, voit en Dracula le traitement littéraire métaphorique du sujet des menstrues. Mais son analyse inédite fait du vampire qui attaque ses victimes et les laisse saignantes, en proie à des pulsions érotiques étouffées ${ }^{31}$, un vecteur approprié pour parler à la fois des menstrues et de la sexualité féminine - un monstre pour mieux parler de deux phénomènes monstrueux : «Bien plus qu'un roman sur les pathologies [...], sa catégorisation du sang masculin comme bon et du sang féminin comme mauvais signale que c'est le sang menstruel et ses pathologies qui provoquent un sentiment d'horreur, [...] mais aussi un hemofétichisme érotisé. Dans le même temps, le roman est un renforcement du point de vue médical conservateur victorien (incarné par des scientifiques comme Icard) selon lequel la menstruation doit être forcément morbide ${ }^{32}$.» Le dégoût de Mina pour son propre corps, ou l'évitement du monstre-Méduse et de son regard pour mieux la décapiter via l'exemple de Lucy, correspondent pour elle à cette volonté de suppression des menstruations comme réponse masculine à la peur du féminin et particulièrement de sa sexualité, qu'elles réveillent.

\section{Mina : femme désirante et désirée}

Selon Gregory Kershner, le personnage littéraire de Mina assume, dans le contexte romanesque d'une Angleterre victorienne érotisée, la fonction intermédiaire d'une 
féminité discréditée - c'est-à-dire l'objet impossible situé à équidistance de la sensualité et de la chasteté. En termes vampiriques, cette fonction devient excès érotique. L'extase orgasmique qu'atteint Mina lorsqu'elle est effectivement attaquée (à laquelle répond la satisfaction toute sadique, et même perverse, complice et voyeuse, de lae lecteurice), est une romantisation du viol, qui se manifeste doublement dans le texte. Il y a la jouissance que ce personnage éprouve à se soumettre aux désirs de Dracula alors même que son discours explicite témoigne de sa répugnance; et la fonction narrative de ces attaques, qui constituent l'un des pics émotionnels les plus forts de l'intrigue. Le récit que Mina fait de ses attaques est à destination d'un auditoire masculin (et de son fiancé d'abord), ce qui opère un déplacement du curseur d'attention, allant de sa peur à leur envie. Entre les lignes, c'est tout l'interdit des violences sexuelles qui est ainsi évoqué : l'angoisse «masculine » de la menstruation est confondue dans le roman avec la peur "féminine » de la première fois/défloration, elle-même une peur déguisée du viol :

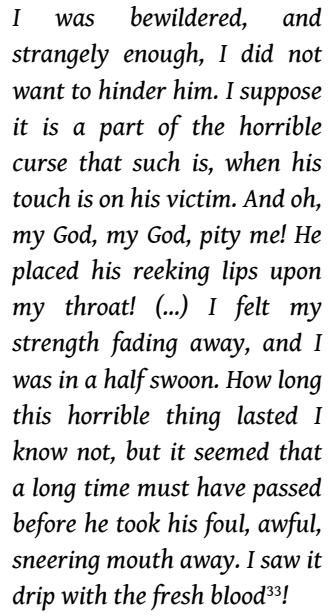

J'étais comme étourdie et, chose étrange, je n'avais nulle envie de m'opposer à son désir. Je suppose que c'est une des conséquences de l'horrible malédiction qui pèse sur ses victimes. Oh! mon Dieu, ayez pitié de moi. C'est alors qu'il colla ses lèvres sur ma gorge. (...) Ma force m'abandonnait de plus en plus, j'étais prête à m'évanouir. J'ignore combien de temps cela dura: mais il me semble qu'il se passa de longs, longs moments avant qu'il ne retirât sa bouche odieuse de laquelle dégoulinait du sang frais ${ }^{34}$.

La continuité éruptive et exubérante du sang et de sa soif en font une métaphore appropriée du désir - un mouvement fluide mais impérieux du sang au-delà des limites corporelles $^{35}$. Cette morsure du vampire, qui intervient au chapitre 21, s'apparente à une agression sexuelle... à laquelle Mina finirait toutefois par prendre du plaisir ${ }^{36}$. Pourtant, Mina manifeste un rejet fort qui s'exprime à la fois dans son discours et son comportement. Après cette attaque, elle s'exclamera à plusieurs reprises "Souillée, souillée! » («Unclean, unclean! ») et refusera que quiconque la touche, tout en refusant elle-même de toucher quoi ou qui que ce soit - ce qui n'est pas sans rappeler certains interdits religieux du Lévitique, dont les prescriptions à l'égard de la menstruation sont particulièrement dures ${ }^{37}$. Cette isotopie de la souillure vampirique/menstruelle, et de la propreté ou pureté contaminée, met en scène le motif de la tache, un canon de représentation récurrent dès qu'il s'agit des fluides, communiquant à la fois la honte des dessous involontairement tâchés, et l'imaginaire des rêves mouillés, ou du drap nuptial à exposer pour prouver par le sang (il est question d'une "red mark» caractéristique) la perte de virginité. Mais la métaphore sexuelle est encore filée et parachevée par ces propos du comte, adressés à Mina au beau milieu de l'acte : 


\begin{tabular}{|c|c|}
\hline $\begin{array}{l}\text { Now you shall come to my } \\
\text { call. When my brain says } \\
\text { 'Come!' to you, you shall cross } \\
\text { land or sea to do my bidding. } \\
\text { And to that end this }{ }^{38 !}\end{array}$ & $\begin{array}{l}\text { Eh bien! Vous devrez désormais } \\
\text { répondre à mon appel. Quand, en } \\
\text { pensée, je vous crierai : « Venez ", } \\
\text { aussitôt vous traverserez terres et } \\
\text { mers pour me rejoindre }{ }^{39} \text { ! }\end{array}$ \\
\hline
\end{tabular}

20 À ce « venez ! » impérieux, la version originale du texte anglais propose le terme come, dont l'ambiguïté est l'un des points signifiants : en effet, et pour tout.e lecteurice contemporain.e, to come signifie aussi jouir, au sens directement sexuel d'avoir un orgasme $^{40}$. L'injonction impérieuse du comte devient donc invitation érotique de l'amant. La double lecture qui en découle colore la suite de l'extrait d'une influence sans équivoque :

\begin{tabular}{|c|c|}
\hline $\begin{array}{l}\text { With that he pulled open his } \\
\text { shirt, and with his long sharp } \\
\text { nails opened a vein in his } \\
\text { breast. When the blood began } \\
\text { to spurt out, he took my hands } \\
\text { in one of his, holding them } \\
\text { tight, and with the other seized } \\
\text { my neck and pressed my mouth } \\
\text { to the wound, so that I must } \\
\text { either suffocate or swallow } \\
\text { some of the... Oh, my God! My } \\
\text { God! What have I done? What } \\
\text { have I done to deserve such a } \\
\text { fate, I who have tried to walk in } \\
\text { meekness and righteousness all } \\
\text { my days. God pity me! Look } \\
\text { down on a poor soul in worse } \\
\text { than mortal peril. And in mercy } \\
\text { pity those to whom she is dear! } \\
\text { Then she began to rub her lips } \\
\text { as though to cleanse them from } \\
\text { pollution }{ }^{41} \text {. }\end{array}$ & $\begin{array}{l}\text { Il déboutonna le plastron de sa } \\
\text { chemise et, de ses mains aux } \\
\text { longs ongles pointus, s'ouvrit } \\
\text { une veine de la poitrine. Lorsque } \\
\text { le sang commença à jaillir, d'une } \\
\text { main il saisit les deux miennes } \\
\text { de façon à me rendre tout geste } \\
\text { impossible, et de l'autre, il me } \\
\text { prit la nuque et, de force, } \\
\text { m'appliqua la bouche contre sa } \\
\text { veine déchirée : je devais donc, } \\
\text { soit étouffer, soit avaler un peu } \\
\text { de... Oh! mon Dieu, qu'ai-je fait } \\
\text { pour devoir endurer tout cela, } \\
\text { moi qui ai pourtant toujours } \\
\text { essayé de marcher humblement } \\
\text { dans le droit chemin? Mon Dieu, } \\
\text { mon Dieu, pitié! Ayez pitié de } \\
\text { mon âme en cet extrême danger, } \\
\text { ayez pitié de ceux qui vous } \\
\text { aiment! } \\
\text { Et elle s'essuya les lèvres, } \\
\text { comme pour les laver de leur } \\
\text { impuretét2. }\end{array}$ \\
\hline
\end{tabular}

21 La multiplication condensée d'allusions sexuelles dans ce seul passage fait de l'échange de sang qui est décrit un échange de fluide autrement plus explicite, que l'auditoire masculin de Mina comprend parfaitement. Les choix de traduction opérés par Jacques Finné mettent de plus l'accent sur le mot veine, lequel terme désigne aussi le phallus en érection - la synecdoque de la " partie désignant le tout » étant on ne peut plus claire : Dracula « se déboutonne », puis ses mains cherchent la veine pour mieux en faire jaillir le fluide ${ }^{43}$.

22 Les appels répétés de Mina à Dieu, tout en rappelant l'ombre pesante de la morale victorienne à laquelle elle s'accroche, pourraient aussi bien manifester les supplications voluptueuses d'une amante au bord de l'extase. L'envie de s'abandonner au comte n'a d'égal que son refus de le faire - une posture à laquelle toute femme peut s'identifier, dans la mesure où les contraintes sociales contradictoires qui pèsent sur la sexualité féminine en font une arme qui peut être retournée contre elle à tout moment pour lui nuire ${ }^{44}$. Une lecture possible des rencontres nocturnes entre Mina et le comte se fait 
ainsi l'écho du fameux «fantasme du viol» très souvent mal compris: si la propre sexualité d'une femme peut être employée contre elle pour la juger ou la condamner, le viol permet de s'abandonner tout en conservant intacte sa pureté, et puisqu'il s'agit d'un pur fantasme, bien différent d'un désir réel, de garder un contrôle total sur les évènements - l'imagination permettant de rêver en toute sécurité. Mais rêve ou fantasme, à l'époque victorienne, il était d'autant plus inconcevable qu'une femme de bonne société puisse aimer le sexe : c'était là l'apanage, croyait-on, des femmes de peu (les prostituées) ${ }^{45}$.

23 Le vampire fait ici de Mina sa complice en la forçant à boire son sang: cet " allaitement » la « marque » ainsi comme son égale, mais aussi comme sa propriété un clin d'œil possible aux mythes de la virginité. La fellation forcée qui est ainsi dite entre les lignes, nous oblige à expliciter le lien direct existant ici avec les théories médicales antiques évoquées précédemment autour des fluides sexuels. Le sang dont il est alors question assume aussi une toute autre fonction par coction : si fellation forcée il $\mathrm{y}$ a, et donc contrainte de boire ce qui apparaît métaphoriquement comme du sperme, il faut se référer à la vision anatomique aristotélicienne selon laquelle le sperme, en se mélangeant au fameux sang menstruel (responsable, nous l'avons dit, des pulsions érotiques de $\mathrm{Mina}^{46}$ ), produirait du lait (et donc insuffle une nouvelle vie). Or, c'est justement là le but de Dracula: assurer à Mina une "nouvelle vie», par la damnation éternelle : métaphore d'un lait nourricier perverti, donné librement pour la transformer - partiellement - en monstre. Cette parodie de communion, de baptême et de mariage (dont la caricature seule, grotesque et horrifique, suffit à choquer la morale victorienne), ne peut donc manquer de nous interpeler. En effet, le fait de boire et de partager du sang tout au long du roman peut tout à fait s'interpréter, selon Marie Mulvey-Roberts, comme une sorte d'allégorie menstruelle liée au sacré et au profane une allégorie qui s'étend elle-même au stigmate menstruel porté par les femmes, par le motif de la «souillure».

24 Enfin, la forme de la blessure de Mina, laissée par le comte au creux de son cou, sera différente des autres, non pas reconnaissable par ces deux piqures habituelles, empreintes des crocs, comme c'est le cas pour Lucy, mais semblable à une seule plaie ruisselante aux bords ourlés, ce qui conduira le personnage de Jonathan, son mari, à s'exclamer terrifié lors de sa découverte : "Au nom du ciel, quel est ce sang? ( «In God's name what does this mean? (...) Mina, dear what is it? What does that blood mean? My God, my God! ${ }^{47}$ »). Là encore, les lèvres rouges et saignantes de la blessure peuvent traduire à la fois l'effarement de la menstruation, ou celui du sang de la défloration. La réaction de Jonathan, révélatrice, prend ainsi un aspect comique, faisant du couple un duo de simples naïfes qui ignorent tout de la réalité sexuelle ${ }^{48}$.

25 Malgré cette attaque, vécue et perçue comme une souillure en dépit de ses connotations sensuelles, Mina obtiendra rédemption : même si elle ne souhaite pas s'y soustraire forcément, Mina a la décence publique d'être horrifiée par ces rencontres (peut-être horrifiée par ses propres sentiments contradictoires), et c'est pour cette raison qu'elle sera sauvée - même si la temporalité de l'attaque correspond avec sa presque disparition de l'intrigue: devenue à son tour ob-scène, elle glisse hors de la scène textuelle et ne remplit plus alors qu'un rôle secondaire, laissant aux personnages masculins le soin d'occuper l'espace narratif. Le monstre rend ainsi visible le fait qu'il est plus acceptable qu'une femme ait été contaminée par un phénomène extérieur à sa volonté, que par une initiative propre : dans les deux cas elle le paye cher (écartement 
narratif/écartement social), mais l'un est une tâche morale un peu moins grave. Car, si ces évènements interviennent toujours contre le gré de Mina, ils permettent toutefois une échappatoire, plutôt que d'accepter l'angoisse suprême: la peur de l'autonomie féminine du désir sexuel, par l'exemple canonique du personnage de Lucy Westenra.

\section{Lucy : femme menstruée, femme monstruée}

26 Face à la chasteté de Mina, Lucy semble le contre-exemple tout désigné. Jeune femme sensuelle, jolie et qui le sait, elle a beaucoup de prétendants et aime à plaire et séduire - ce qui fait de son caractère une personnalité frôlant déjà dangereusement les limites de ce qui est acceptable pour une femme. Ainsi, tout en voyant en Méduse un symbole approprié du dénigrement des mystères corporels féminins sacrés autour des menstruations ("menstruation-based sacred female mysteries»), Mary Mulvey-Roberts explique en quoi Dracula pourrait être une relecture de l'histoire même du cycle menstruel. Le complexe de castration souvent évoqué par Freud à propos de Méduse (les serpents étant une manière appropriée de déplacer le symbole phallique pour adoucir l'horreur) est ainsi retourné : tout comme une castration, la menstruation induit un saignement génital angoissant, qui apparait en outre comme « magique » car ne déclenchant pas de souffrance chez la femme ni n'entrainant sa mort. Le pouvoir attribué à la menstruation suggère la place qu'elle occupe au centre d'un système où la morsure du vampire en devient le punctum signifiant.

27 Tout comme Mina, Lucy va être mordue, mais les conséquences de cette attaque seront pour elle bien différentes : son comportement, devenu vorace, et son appétit insatiable (qui se confond avec un appétit sexuel dévorant), ne sont pas sans rappeler les sbires féminins du château du comte. Contrairement à Mina, Lucy sera transformée par Dracula, ce qui fait d'elle une victime non plus seulement par contact, mais par contamination ou assimilation - dans le roman, cela se traduit notamment par la transformation de ses cheveux: de blonds, ils deviennent noirs, symbole d'une innocence corrompue et d'une monstruosité émergente ${ }^{49}$.

28 Le destin tragique de Lucy est le miroir du rôle assigné aux femmes et de la sanction sociale immédiate et définitive qui s'ensuit si elles dérivent de la norme : à l'instar des utopies positivistes évoquées précédemment au sujet de la prostitution, le sacrifice de Lucy sera un mal nécessaire.

29 Les personnages principaux masculins vont ainsi décider que Lucy mérite une punition exemplaire, laquelle fait un écho sinistre aux remèdes à l'hystérie qui témoignaient, comme on le sait, d'un sadisme sexuel inouï. Ainsi, au moment où le personnage de Lucy présente les premiers symptômes de "l'hystérie » et des troubles menstruels notés par ces médecins victoriens influencés par cette préconception de désordre des fluides, elle est à un point déterminant de son développement sexuel, mais c'est aux visites nocturnes de Dracula qu'il faut imputer son exacerbation, faisant d'elle la première victime non pas du comte, mais de la morale répressive de ceux qui sont perçus comme ses sauveurs - dont son propre fiancé, Arthur. Or, la "punition » en question, n'est autre que sa mise à mort. Lucy va être traitée par ce groupe entièrement masculin comme le monstre femelle par excellence, Méduse, archétype qui, si l'on se rappelle les lectures freudiennes, et plus tard celles, critiques, de Cixous, se trouve justement être une métaphore du sexe féminin plus particulièrement encore du sexe féminin pendant les règles : 
Arthur took the stake and the hammer, and when once his mind was set on action his hands never trembled nor even quivered. Van Helsing opened his missal and began to read, and Quincy and I followed as well as we could. Arthur placed the point over the heart, and as I looked I could see its dint in the white flesh. Then he struck with all his might.

The Thing in the coffin writhed; and a hideous, blood-curdling screech came from the opened red lips. The body shook and quivered and twisted in wild contortions; the sharp white teeth champed together till the lips were cut, and the mouth was smeared with a crimson foam. But Arthur never faltered. He looked like a figure of Thor as his untrembling arm rose and fell, driving deeper and deeper the mercy-bearing stake, whilst the blood from the pierced heart welled and spurted up around it. His face was set, and high duty seemed to shine through it; the sight of it gave us courage, so that our voices seemed to ring through the little vault.

And then the writhing and quivering of the body became less, and the teeth ceased to champ, and the face to quiver. Finally it lay still. The terrible task was over [...]

Arthur bent and kissed her, and then we sent him and Quincey out of the tomb; the Professor and I sawed the top off the stake leaving the point of it in the body. Then we cut off the head and filled the mouth with garlic ${ }^{50}$.
Arthur prit le pieu et le marteau, et une fois qu'il eut fermement décidé son esprit à agir [une métaphore effrayante du devoir], ses mains ne tremblèrent pas le moins du monde, n'hésitèrent même pas. Van Helsing ouvrit le missel, commença à lire; Quincey et moi lui répondîmes de notre mieux. Arthur plaça la pointe du pieu sur le cœur de Lucy, et je vis qu'elle commençait à s'enfoncer légèrement dans la chair blanche. Alors, avec le marteau, Arthur frappa de toutes ses forces.

La Chose dans le cercueil sursauta, et un horrible cri, propre à vous glacer le sang, s'échappa des lèvres rouges [nous soulignons]. Le corps s'agita et se tordit en d'horribles contorsions; les dents acérées s'enfoncèrent dans les lèvres au point de les couper, et elles se couvrirent d'une écume écarlate. Mais, à aucun moment, Arthur ne perdit courage. Il ressemblait au dieu Thor tandis que son bras ferme s'élevait et retombait, enfonçant de plus en plus le pieu miséricordieux, et que le sang jaillissait du cœur percé et se répandait tout autour. $\mathrm{La}$ résolution était peinte sur son visage, comme s'il était certain d'accomplir un devoir sacré et, à le voir, nous ne nous sentions que plus de courage, de sorte que nos voix, plus fortes, résonnaient maintenant dans le caveau.

Peu à peu, le corps cessa de trembler, les contorsions s'espacèrent, mais les dents continuaient à s'enfoncer dans les lèvres, les traits du visage à frémir. Finalement, ce fut l'immobilité complète. La terrible tâche était terminée. [...]

Arthur se pencha et déposa un baiser sur le visage paisible. Puis nous les fimes sortir du tombeau, Quincey et lui. Alors, j'aidai le professeur à scier le haut du pieu, laissant la pointe enfoncée dans le corps. Puis, nous coupâmes la tête et remplîmes la bouche d'ail ${ }^{51}$.

30 Dans cet extrait, le parallèle qui peut être fait entre le pieu perforant le cœur/corps de Lucy, et les méthodes utilisées pour soigner l'hystérie (comme enfoncer des bâtons ou chiffons dans le vagin des femmes), « soins » qui ont de plus été réalisées en public par les praticiens, font de cette scène en particulier une scène très "charcotienne ». L'empalement peut bien sûr figurer une pénétration qui « règle » le «problème 
féminin »; et la décapitation renvoyer, in fine, aux clitoridectomies pratiquées sur des hystériques et autres «maniaques » sexuelles. C'est ainsi précisément la lecture qu'en fait le critique Darryl Jones ${ }^{52}$ : cette mise à mort commune de Lucy par un «public » d'hommes actifs, c'est l'évocation d'un viol collectif - un viol punitif qui fait de la bouche saignante une image de sexe violenté beaucoup plus graphique. Cette interprétation fait ainsi tristement écho aux viols correctifs avec lesquels on traitait l'hystérie - «l'empalement» par plusieurs hommes prend donc ici une tournure symbolique particulièrement angoissante ${ }^{53}$.

31 L'histoire personnelle de Lucy peut aussi être comprise comme un exemple de la femme déchue, qui a transgressé les codes de ce que la morale de son époque jugeait décent pour une femme, et en a payé le prix. C'est ainsi une représentation de la supposée insanité que la menstruation déclenche chez les femmes, notamment ses conséquences sur la sexualité, en accord avec l'idée selon laquelle « sous la lumière de la lune, cette force féminine enragée et affamée est libérée ${ }^{54}$ ». Lorsqu'elle est empêchée par Van Helsing de tenter son fiancé avant d'être tuée, la fureur de Lucy prend des proportions gorgonesques. Sa bouche rouge dégoulinante de sang est une métaphore vivante du sexe saignant pendant les menstrues. La scène s'achève par la reprise de contrôle dudit fiancé sur le phénomène, qui lui plante un pieu et échappe à ce vagina dentata, avant que Van Helsing, tel un Persée vengeur, ne décapite Lucy (un sort funeste qu'elle a ainsi en partage avec Méduse).

32 Comme Maria Parsons, dans son article « Vamping the Woman: Menstrual Pathologies in Bram Stoker's Dracula ${ }^{55} »$, on peut arguer que le vampire, dans le roman de Stoker, sert à déplacer cette incarnation de la peur masculine des menstruations à la peur masculine de la sexualité féminine, ce qui permet de révéler les stratifications de pouvoir contenues dans les interactions entre les discours médicaux et moraux de l'époque autour du cycle menstruel. Parsons voit en Lucy l'exemple même des angoisses socioculturelles et psychosexuelles liées à la menstruation, et de leurs ravages sur les femmes. "De sa première rencontre avec Dracula jusqu'à sa décapitation finale et son empalement, Lucy est une étude de cas exemplaire de la pathologisation de la menstruation, et du contrôle et du confinement de la sexualité féminine ${ }^{56}$ ", affirme-t-elle. Lucy, qui souffre de son rôle limité de "femme " tel que le concevait la société victorienne, n'a pas d'autre choix que de supprimer tout désir d'explorer sa sexualité et est obligée de remplir son devoir. Cette abnégation masochiste est sa seule option (et la seule réservée aux femmes) dans une société qui nie vigoureusement toute expression de la sexualité et du désir féminins. Pour Parsons, le destin de Lucy, tout comme les traitements qu'elle subira (des transfusions sanguines récurrentes notamment) mettent en évidence l'obsession victorienne qui consiste à traiter les maladies mentales féminines - comme pouvait l'être un désir sexuel trop exprimé - en cherchant d'abord à réguler le cycle menstruel. La Lucy de Stoker est une image vibrante de la maturité sexuelle féminine. Cependant, le châtiment qu'elle encourra pour avoir laissé s'exprimer ses pleins besoins est cauchemardesque: la bouche encore rouge de sang frais, elle sera donc empalée puis décapitée.

\section{Conclusion}

Dans Dracula, les angoisses sont à la fois de nature circulatoire (liées aux fluides corporels), et profondément sexuelles. Cette «mécanique des fluides » est ainsi mise en 
perspective par l'écriture, notamment du point de vue de ses incidences sur la vie sociale et les représentations symboliques des femmes. Pour répondre au trouble engendré par l'apparition des différents fluides sexuels, la gestion dans le texte des diverses circulations revient à pérenniser le modèle socio-économique bourgeois (capitaliste et libéral) de la société victorienne - dont le roman, à travers le choix de ses personnages, peut se faire l'écho. Il est alors juste de dire que le discours patriarcal en vigueur, notamment médical, s'enracine aussi dans un contexte social, politique et économique singulier.

34 Le gothique horrifique et macabre du XIX ${ }^{\mathrm{e}}$ siècle sert ici une fonction bien définie : celle d'exorciser les peurs fantasmées de débordement moral, et de la contagion par impureté d'une certaine classe sociale dominante. Ce que Dracula met en scène, ce sont donc les peurs et les valeurs d'une société à la veille de son effondrement - exemplifié, en particulier, par la barbarie ridicule de la pseudo-science positiviste établissant des discours autour des phénomènes circulatoires, plus particulièrement sexuels, dans un souci d'hygiène sociale et morale. Ainsi, l'exemple plus spécifique du phénomène menstruel, et son lien à l'hystérie, qui serait à l'origine d'une sexualité féminine diabolique et vorace, se fait le vecteur critique privilégié des discours victoriens abondant sur la question de ce qu'est, ou devrait être, la sexualité : la vision de la sexualité et de l'hypersensibilité féminines qu'il véhicule offre ici une critique de la "nécessaire» action des praticiens masculins pour maîtriser le corps et le comportement des femmes - jeu de pouvoir et de domination. Tout ceci fait donc du roman du vampire sanguinaire, éminemment licencieux et « horrifique » par les grands bouleversements qu'il met en scène - sociaux, médicaux, genrés -, une formidable étude de cas des discours victoriens entourant la sexualité, dont les fluides corporels révèlent le trouble. Le contrôle et la répression s'y traduisent par la lutte contre le monstre vampirique, incarnation de la menace venue des marges. Ainsi, le roman de Stoker, refusant la circulation sage, exacerbe au contraire le débordement tant des angoisses liées à ces préoccupations... que des fluides qui en résultent.

\section{BIBLIOGRAPHIE}

ARISTOTE. 1961. De la génération des animaux, traduction de Pierre Louis, Paris : Les Belles Lettres. ARNAUD Sabine. 2014. L'invention de l'hystérie au temps des Lumières (1670-1820), Paris : EHESS.

AYDEMIR Mourat. 2007. Images of Bliss : Ejaculation, Masculinity, Meaning, Minneapolis, Minnesota : University of Minnesota Press.

BACHELARD Gaston. 1942. L'eau et les rêves. Essai sur l'imagination de la matière, Paris : Le Livre de Poche, éd. José Corti.

BERTHET Dominique. 2015. Une esthétique du trouble, Paris : L’Harmattan.

BERTHET Dominique. 2012. Recherches en Esthétique n 17 : « Le trouble », CEREAP.

BOTTING Fred. 1995. Gothic, coll. « The Nex Critical Idiom », London : Routledge. 
BUTLER Judith. 2006 [1990]. Trouble dans le genre, trad. Cynthia Kraus, Paris : La Découverte. DIDEROT Denis. 1980 [1769] Le rêve de d'Alembert, in Euvres complètes, vol. 17 (éd. Jean Varloot), Paris : Hermann.

DIJKSTRA Bram. 1992. Idols of perversity, Oxford: Oxford University Press.

DELANEY Janice, LUPTON Mary-Jane \& TOTH Emily. 1988. The Curse : A Cultural History of Menstruation, Urbana, Chicago : University of Illinois Press.

DERRIDA Jacques. 1998. Voiles, Paris : Galilée

D'ONOFRIO Salvatore. 2014. Les Fluides d'Aristote. Lait, sang et sperme dans l'Italie du Sud, Paris : Les Belles Lettres.

DORLIN Elsa. 2006. Matrice de la race, Paris : La Découverte.

DOUGLAS Mary. 2005 [1966]. De la souillure. Essai sur les notions de pollution et de tabou, trad. Anne Guérin, Paris : La Découverte.

GITTER Elisabeth G. 1984. « The Power of Women's Hair in the Victorian Imagination », PMLA Vol. 99, No. 5.

HANAFI Nahema, 2017, Le frisson et le baume : expériences féminines du corps au Siècle des Lumières, Rennes : Presses universitaires de Rennes.

HERITIER Françoise. 1966. Masculin/Féminin, La pensée de la différence, Paris : O. Jacob.

KERSHNER Gregory. 2006. « Horror \& Eroticism : Bram Stoker's Dracula », in HOFSTRA Horizons. JONES Darryl. 2002. Horror: A Thematic History in Fiction and Film, London : Arnold.

KOLNAI Aurel. 1997. Le dégoût, trad. Olivier Cossé, Paris : Agalma.

KRISTEVA Julia. 1980. Pouvoirs de l'horreur, Paris : Seuil.

LAHUERTA Claire. 2011. Humeurs. L'écoulement en art comme herméneutique critique du corps défaillant, Paris : L'Harmattan.

LAQUEUR Thomas. 2013 [1990]. La Fabrique du sexe, trad. Michel Gautier, Paris : Gallimard.

LAW Jules David. 2010. The Social Life of Fluids, Cornell: Cornell University Press.

LEVI-STRAUSS Claude. 1978. Le cru et le cuit, Paris : Plon.

LOMBROSO Cesare. 1887. L'homme criminel, Étude anthropologique et psychiatrique, trad. Régnier et Bournet, Paris : Félix Alcan.

MULVEY-ROBERTS Marie. 2005. «Menstrual Misogyny and Taboo: The Medusa, Vampire and the Female Stigmatic », in Menstruation: A Cultural History, Londres : éd. Gillian Howie, Andrew Shail. PARENT DU CHATELET Alexandre. 2010 [1857]. De la prostitution dans la ville de Paris, Paris : Nabu Press.

PARSONS Maria. « Vamping the Woman: Menstrual Pathologies in Bram Stoker's Dracula », in The Irish Journal of Gothic and Horror Studies, consulté en ligne le 13/04/16: http:// irishgothichorrorjournal.homestead.com/maria.html.

QUIGNARD Pascal. 1996. Le sexe et l'effroi, Paris : Folio.

RENNES Juliette (dir.). 2016. Encyclopédie critique du genre. Corps, sexualité, rapports sociaux, Paris : La Découverte. 
RINGROSE Jessica. 2012. Postfeminist Education? : Girls and the Sexual Politics of Schooling, Londres : Routledge.

STOKER Bram. 2015 [1897]. Dracula, Milton-Keynes : Penguin Classics, Penguin English Library.

STOKER Bram. 2013 [1897], Dracula, traduction de Jacques Finné, Paris : Pocket.

\section{NOTES}

1. C'est en effet notre conviction que la manipulation esthétique et symbolique des fluides a pour rôle la métamorphose cognitive de la relation que nous entretenons avec eux, laquelle n'est possible que par rapport à l'inconnu, à l'autre, qu'à partir de la défiguration, de la défamiliarisation, de l'« inquiétante étrangeté » - en somme, du trouble. Le trouble est ce qui fait désordre et crée un écart, ce qui bouscule les normes : nous déréglons un prédicat, une certaine qualité, nous sortons de la règle et, ce faisant, nous agitons et émouvons le système entier. Dans le cas des fluides corporels, il en va de l'expression "violente ", c'est-à-dire excessive, voire obscène, du corps humain (Voir BATAILLE Georges. 1982. L'Érotisme, Paris : Minuit). Censés être contenus par l'enveloppe de la chair et de la peau, subsumés à une intériorité cachée (au sens littéral du terme), ces fluides deviennent, en s'extériorisant, un surplus ou un foisonnement vital incongru, qui dérange et angoisse. C'est ainsi à une écriture double, de l'absence/présence, de la limite, du choc esthétique, en même temps qu'à une écriture parfois violente de la matière et de la matérialité du corps ouvert, obscène, intime, que nous invite la réalité de ces produits et excrétions, et les gestes esthétiques qui les exploitent.

2. Aristote postule en effet, dans De la génération des animaux (1961. traduction de Pierre Louis, Paris : Les Belles Lettres.), une nature commune entre sperme et menstrues, puis entre menstrues et lait. Durant l'acte sexuel, le mélange supposé du sperme et du sang menstruel résulterait en la formation du lait nourricier. La tradition médicale qui découle des observations d'Aristote fonde ce rapprochement reliant directement le sperme fécondateur au sang menstruel : on pourrait ainsi déduire, par extension, une éventuelle nature commune entre sperme et lait.

3. Voir RENNES Juliette (dir.). 2016. Encyclopédie critique du genre. Corps, sexualité, rapports sociaux, Paris : La Découverte.

4. D'ONOFRIO Salvatore. 2014. Les Fluides d'Aristote. Lait, sang et sperme dans l'Italie du Sud, Paris : Les Belles Lettres.

5. DERRIDA Jacques, Voiles, Galilée, Paris, 1998, p.45.

6. BERTHET Dominique. 2015. Une esthétique du trouble, Paris : L'Harmattan ; et 2012. Recherches en Esthétique $\mathrm{n}^{\circ} 17$ : « Le trouble », CEREAP.

7. "The blood of the covenant is thicker than the water of the womb. ", expression britannique, sans direct équivalent en français, que l'on pourrait traduire par « Le sang de l'alliance est plus dense que les eaux de la matrice. ». L'usage courant raccourci de cette citation en langue anglaise, que nous utilisons ici comme titre, signifie que les liens du sang sont communément acceptés comme étant les plus forts qui existent; mais le sens véritable de ce proverbe semble suggérer au contraire que le sang versé entre frères d'armes aurait plus de poids que les liens familiaux. Quoi qu'il en soit, dans la version abrégée ou complète de cette expression, il est clair que le sang revêt plus d'importance que l'eau.

8. Gaston Bachelard affirmait que, pour l'imagination, tout ce qui coule est de l'eau. (1942. L'eau et les rêves. Essai sur l'imagination de la matière, Paris : Le Livre de Poche, éd. José Corti.) Or, le contrôle de l'eau est un enjeu essentiel de l'industrialisation, même si l'on peine à se déprendre des angoisses selon lesquelles les eaux font circuler les maladies. Cette angoisse de circulation transparait dans la littérature victorienne, qui la transpose à des romans comme Dracula mettant abondamment en scène d'autres formes de circulation. Il y a là un discours sanitaire, qui est aussi 
un discours plus directement social sur les rôles propres à chacun - du point de vue de la classe comme du genre. Il s'opère alors un déplacement signifiant de cet enjeu : si l'on parvient à réguler et donc contrôler la circulation de la Tamise, notamment via une arrivée d'eau dans les foyers, on peut réguler les flux de la circulation sociale. C'est ainsi la lutte entre les réclamations de l'individu et les réclamations de la collectivité qui s'exprime. Voir également LAW Jules David. 2010. The Social Life of Fluids, Cornell: Cornell University Press.

9. Françoise HERITIER (1966. Masculin/Féminin, La pensée de la différence, Paris : O. Jacob) l'explique ainsi : le tabou des fluides fait écho, d'un point de vue anthropologique, aux tabous du corps, et $\mathrm{du}$ corps féminin en particulier - $\mathrm{du}$ fait même de ce lien entre féminité et fluidité précédemment évoqué . Nombreux sont donc les discours existant sur le rôle des fluides qui semblent appuyer la justification de la valence différentielle des genres et de la domination masculine. Voir également BUTLER Judith. 2006 [1990]. Trouble dans le genre, trad. Cynthia Kraus, Paris : La Découverte.

10. Voir DORLIN Elsa. 2006. Matrice de la race, Paris : La Découverte.

11. Voir HANAFI Nahema, 2017, Le frisson et le baume : expériences féminines du corps au Siècle des Lumières, Rennes : Presses universitaires de Rennes.

12. Aristote parle ainsi d'un " défaut de chaleur vitale de perfection », op. cit., II, 4, p.17.

13. LAQUEUR Thomas. 2013 [1990]. La Fabrique du sexe, trad. Michel Gautier, Paris : Gallimard.

14. DIDEROT Denis. 1980 [1769] Le rêve de d'Alembert, in Cuvres complètes, vol. 17 (éd. Jean Varloot), Paris : Hermann, p. 152 : «La femme est le premier monstre de l'homme.».

15. Claire Lahuerta (2011. Humeurs. L'écoulement en art comme herméneutique critique du corps défaillant, Paris : L'Harmattan.) parlera d' « herméneutique de la défaillance » pour caractériser l'angoisse particulière générée par la charge affective et symbolique des fluides qui se répandent. 16. DOUGLAS Mary. 2005 [1966]. De la souillure. Essai sur les notions de pollution et de tabou, trad. Anne Guérin, Paris : La Découverte, p.61.

17. Ibid., p. 137.

18. BOTTING Fred. 1995. Gothic, coll. «The Nex Critical Idiom », London : Routledge.

19. Mourat AYDEMIR (2007. Images of Bliss: Ejaculation, Masculinity, Meaning, Minneapolis, University of Minnesota Press, Minnesota) parle de "force éjaculatrice de l'oeil".

20. Le rapport de l'individu aux fluides est un rapport extrêmement complexe. Il existe on le sait une répugnance anthropologique du mou par rapport au dur, du cru par rapport au cuit... et du fluide, du liquide, par rapport au solide (dichotomie informe/forme définie) - voir LEVI-STRAUSS Claude. 1978. Le cru et le cuit, Paris: Plon. De tous les composants du corps, les fluides de la différence sexuelle, sans doute par leur caractère informe, mais plus probablement du fait de diverses constructions et représentations culturelles, sont les plus déconsidérés et méprises de tous les fluides, tout au moins d'un point de vue strictement occidental - ce qui indique une première borne à cet angle de vue. Ainsi, si les fluides corporels en général sont par définition des matières ignobles, indignes, des déchets humiliants qui nous rattachent à la fois aux contingences matérielles et à une certaine animalité, les fluides du sexe sont témoins ouvertement révélateurs d'une activité que l'être humain s'est efforcé de policer pour en contenir la portée : la sexualité, ses antécédents et ses conséquences. Sperme, lait et menstrues, représentent par conséquent une tension, un trouble, de possibles lieux de pouvoir et réservoirs sémantiques où quelque chose se joue, précisément en vertu de leur aptitude à évoquer dans leur réalité crue une activité cachée qui échappe toujours, en définitive, au contrôle social et à la norme. En effet, lorsque tant Aurel Kolnai que Julia Kristeva (1980. Pouvoirs de l'horreur, Paris : Seuil.) établissent respectivement une catégorisation ou nomenclature des " objets dégoûtants " physiques, iels n'hésitent pas à prendre en compte les secrétions du corps comme objets de répugnance avérée. Ce dégoût du visqueux et du caractère excrémentiel des fluides est ainsi renforcé par les interdits et connotations spécifiques qui leur sont liés. 
21. KOLNAI Aurel. 1997. Le dégoût, trad. Olivier Cossé, Paris : Agalma, p.47-48 : « On peut tenir pour assuré que l'objet du dégoût [parmi lesquels il classe les secrétions] manifeste une propension au secret, à la stratification, à l'impénétrabilité, à l'inquiétant, aussi bien qu'à l'impudeur, à l'importunité et à la séduction ou tentation. (L'anglais taunting exprime le mieux cette nuance.) Tout ce qui est dégoûtant a quelque chose de frappant et d'énigmatique à la fois, analogue à une baie rouge et vénéneuse ou à un maquillage criard. »

22. STOKER Bram. 2015 [1897]. Dracula, Milton-Keynes : Penguin Classics, Penguin English Library, p.56.

23. STOKER Bram, 2013 [1897], Dracula, traduction de Jacques Finné, Paris : Pocket, p.106.

24. Au-delà de l'hystérie et de son lien avec un appétit sexuel vorace, déplacé chez une femme, et sur lequel nous reviendrons, il importe de souligner que le diagnostic de la fureur utérine, ou nymphomanie, est tout aussi prégnant à cette époque.

25. Op. cit., p.56-57.

26. Op.cit. , p.107.

27. KERSHNER Gregory. 2006. "Horror \& Eroticism: Bram Stoker's Dracula ", in HOFSTRA Horizons.

28. DIJKSTRA Bram. 1992. Idols of perversity, Oxford: Oxford University Press.

29. ARNAUD Sabine. 2014. L'invention de l'hystérie au temps des Lumières (1670-1820), Paris : EHESS.

30. DELANEY Janice, LUPTON Mary-Jane \& TOTH Emily. 1988. The Curse: A Cultural History of Menstruation, Urbana, Chicago : University of Illinois Press.

31. Même si le mythe vampirique procure comme on le sait à ce phénomène une explication " rationnelle », la nuit constitue, d'un point de vue littéraire et imaginaire, une marge en soi. C'est dans les ténèbres que les monstres vivent et opèrent, mais également dans les ténèbres que les actes relevant de l'«ob-scène", qui ne peuvent avoir lieu en pleine lumière, trouvent naturellement leur place. La nuit est donc un espace propre, et une temporalité codifiée, connotée, réservée à l'intime et au secret. Voir également QUIGNARD Pascal. 1996. Le sexe et l'effroi, Paris : Folio.

32. MULVEY-ROBERTS Marie. 2005. «Menstrual Misogyny and Taboo: The Medusa, Vampire and the Female Stigmatic ", in Menstruation: A Cultural History, Londres : éd. Gillian Howie, Andrew Shail, p.159 : «Far more than a novel about pathologies. [...] its gendering of male blood as good and female blood as bad signals that it is menstrual blood and its pathologies that provoke a sense of horror. [...] Stoker's attention to the relationship between women and blood is a surrogate for menstrual taboo, which is also eroticized haemofetishism. At the same time, it is a reinforcement of the Victorian conservative medical view that menstruation should be morbidified.. » (Traduction libre)

33. STOKER Bram, op. cit., p. 412

34. STOKER Bram, op. cit., p.306

35. Voir KERSHNER Gregory, op. cit.

36. Parmi les mythes existant autour du viol, on retrouve cette idée persistante que le désir féminin est une chose trouble et mystérieuse, dont l'action coercitive maintient le consentement sexuel des femmes dans une «zone grise» dialectique que le roman met parfaitement en évidence.

37. «Lorsqu'une femme a un écoulement de sang et que du sang s'écoule de son corps, elle restera pendant sept jours dans la souillure de ses règles. Qui la touchera sera impur jusqu'au soir. Toute couche sur laquelle elle s'étendra ainsi souillée, sera impure ; tout meuble sur lequel elle s'assiéra sera impur. Quiconque touchera son lit devra nettoyer ses vêtements, se laver à l'eau, et il sera impur jusqu'au soir. Quiconque touchera un meuble, quel qu'il soit, où elle se sera assise, devra nettoyer ses vêtements, se laver à l'eau, et il sera impur jusqu'au soir. » Lv 15:19-22

38. Ibid., p.413

39. Ibid. 
40. Détail par ailleurs intéressant, l'argot britannique ("slang») dévie de «to come » le verbe « to cum », signifiant « éjaculer».

41. Ibid.

42. Ibid.

43. Plus particulièrement, "faire un aller retour sur la veine bleue » signifie "faire une fellation »: on peut donc imaginer ce que «ouvrir la veine» peut vouloir dire en double sens (phonesthème des « vannes », également à ouvrir ?).

44. C'est ici toute la conceptualisation féministe du "slut-shaming " ou "slut-bashing ». Voir RINGROSE Jessica. 2012. Postfeminist Education? : Girls and the Sexual Politics of Schooling, Londres : Routledge, p. 93.

45. On considérait que seules les prostituées aimaient le sexe, et que la prostitution était, selon le théoricien Parent-Duchâtelet, un "égoût séminal » nécessaire (on notera l'expression) pour lequel il était acceptable et même justifié qu'une certaine frange de la population féminine soit sacrifiée (mythe de la prostituée née pour cela, faite pour cela et qui aime cela: un ressort apprécié du positivisme scientifique mis en scène par Stoker et exemplifié par les travaux physiognomoniques du docteur Lombroso sur le concept de " prostituée-née »). Voir PARENT DU CHATELET Alexandre. 2010 [1857]. De la prostitution dans la ville de Paris, Paris : Nabu Press; et LOMBROSO Cesare. 1887. L'homme criminel, Étude anthropologique et psychiatrique, trad. Régnier et Bournet, Paris : Félix Alcan.

46. En effet, une toute autre lecture pourrait voir en cette première attaque sanglante mais sexuelle du monstre une métaphore de la « première fois », laquelle pourrait n'être autre qu'une provocation contrôlée de la menstruation.

47. STOKER Bram, Dracula, op. cit., p. 406.

48. Voir également MULVEY-ROBERTS Mary, op. cit.

49. Dans son étude « The Power of Women's Hair in the Victorian Imagination » (1984. PMLA Vol. 99, No. 5, p. 936-954), Elisabeth G. GITTER écrit: « The more abundant the hair, the more potent the sexual invitation implied in its display. For folk, literary and psychoanalytic traditions agree that the luxuriance of the hair is an index of vigorous sexuality, even of wantonness. » (p. 938)

50. STOKER Bram, Dracula, op.cit., p.307-309.

51. STOKER Bram, Dracula, op. cit., p.234.

52. JONES Darryl. 2002. Horror: A Thematic History in Fiction and Film, London : Arnold, p.88.

53. Voir par exemple l'article du 02/03/16, consacré à ce sujet, du Huffington Post: http:// www.huffingtonpost.fr/2016/02/29/francais-victime-viol-etude_n_9346702.html (consulté le 11/05/15)

54. MULVEY-ROBERTS Mary, op. cit., p.155 : «Under the light of the moon this raging, hungry female force is released. » (Traduction libre)

55. Maria PARSONS, «Vamping the Woman: Menstrual Pathologies in Bram Stoker's Dracula ", in. The Irish Journal of Gothic and Horror Studies, consulté en ligne le 13/04/16 : http:// irishgothichorrorjournal.homestead.com/maria.html.

56. Ibid. : «From her first encounter with Dracula to her final beheading and staking, Lucy is an exemplary case study in the pathologising of menstruation and the control and containment of female sexuality. » (Traduction libre) 


\section{RÉSUMÉS}

La figure du monstre vampirique dans Dracula ex-prime une menace venue des marges. Or, ces marges ont une inscription résolument corporelle. En partant des préoccupations angoissées, toutes victoriennes, persistant à l'égard de la circulation, on s'interrogera sur la visibilité des fluides « du sexe» (sperme, menstrues et lait) dans le roman. L'article démontrera que la «mécanique des fluides» ainsi pensée par Bram Stoker se pose en des termes simples: une esthétique du trouble, où le débordement qui s'incarne, notamment dans les personnages féminins, remplit une fonction cathartique.

The vampiric monster in Stoker's Dracula epitomizes a threat coming from the margins, which happens to be related to the body. Indeed, anxious Victorian concerns persisting about different types of flow lead us to question the concrete visibility of sexual bodily fluids (sperm, menses and milk) in the novel. This study will hence discuss how Stoker's "fluid mechanics" conveys an "aesthetics of trouble", and how its overflowing serves a cathartic purpose with a direct link to the writing of female protagonists.

INDEX

Thèmes : Recherches

Keywords : Dracula, bodily fluids, trouble, blood, sperm

Mots-clés : Dracula, fluides corporels, trouble, sang, sperme

\section{AUTEUR}

\section{MAËL BAUSSAND}

Maël Baussand est photographe plasticienne et rédactrice pour le magazine politique Simonae. Elle prépare actuellement une thèse de doctorat en littérature comparée sur les fluides corporels : "Sécrétions Magnifiques : une esthétique du trouble »; sous la direction de Pierre Zoberman et Anne-Isabelle François. Elle est rattachée à l'Université Paris 3 Sorbonne-Nouvelle (ED 120, CERC, EA 172). 